\title{
A Hardware-Oriented Method for Evaluating Complex Polynomials
}

\author{
Miloš D. Ercegovac \\ Computer Science Department \\ University of California at Los Angeles \\ Los Angeles, CA 90024, USA \\ milosecs.ucla.edu
}

\author{
Jean-Michel Muller \\ CNRS-Laboratoire LIP, projet Arénaire \\ Ecole Normale Supérieure de Lyon \\ 69364 Lyon Cedex 07, FRANCE \\ Jean-Michel.Mullerdens-lyon.fr
}

\begin{abstract}
A hardware-oriented method for evaluating complex polynomials by solving a system of corresponding linear equations is proposed. It is based on the E-method [2, 3], defined over reals, which uses efficient digit-serial solution of diagonally dominant systems of linear equations on a simple and highly regular hardware. Since the evaluation of polynomials can be achieved by solving the corresponding linear systems, the E-method is an attractive general approach for polynomial evaluation. We show a transform of the E-method to the complex domain, describe the complex polynomial evaluation algorithm, and discuss a corresponding design and implementation. We give estimates of the latency and the area.
\end{abstract}

\section{Introduction}

In this paper we describe a new method for evaluation of complex polynomials, suitable for hardware implementation. It has a latency of $m$ cycles for $m$-bit precision and a repetitive implementation which corresponds roughly to $n+1$ serial-parallel multipliers for polynomials of degree $n$. The coefficients and argument are fixed-point complex numbers. The proposed method is a generalization of a polynomial evaluation method over reals introduced as the E-method [2, 3], and recently overviewed in [7]. This paper is based on the report [8] where the complex E-method is introduced and discussed in more general terms.

The E-method is a two-phase approach: a polynomial is mapped a digit-iterative method for solving systems of linear equations, the E-method $[2,3,7]$, to allow the use of the complex number system. The proposed approach is suitable for hardware implementation. The main characteristics of the method are: (i) $m$-digit solution is computed in about $m$ steps, each step consisting of a sum of numberby-digit products, (ii) the cycle time depends on the number of nonzero coefficients, (iii) the cycle time does not de- pend on the precision $m$ (if redundant additions are used), (iv) for a system of order $n$, the shortest latency requires $n$ elementary units for the real part, and $n$ units for the imaginary part, and (v) the elementary units are interconnected with digit-wide links. The approach is particularly efficient when the coefficient matrix is sparse. This happens when the E-method is used to evaluate polynomials (one off-diagonal element) and rational functions (two offdiagonal elements). Other examples are a tridiagonal system (two off-diagonal elements), powers of the argument (one off-diagonal element), and special expressions.

We first introduce the transform which allows the Emethod to be used in the complex field $\mathbf{C}$. Then we show how to use the complex E-method (CE-method) in evaluating complex polynomials as a particularly interesting case. Evaluation of consecutive powers of a complex argument is a special case of polynomial evaluation and can be performed on the same hardware.

Complex polynomials appear in many areas such as digital signal and image processing, control systems, and applied mathematics, in general. A Horner type method for evaluating complex polynomials is proposed in [1] at the algorithm level, implicitly assuming a software implementation. The method uses $O(n)$ multiplications and $O(n)$ additions for a complex polynomial of degree $n$. If these multiplications and additions are performed in a sequential order, the latency of the method is about $n \times T_{M U L T-A D D}$ which is significantly slower than our method. If a parallel algorithm for polynomial evaluation is used, the total time is about $\log n \times T_{M U L T-A D D}$ which is still slower than our method. The evaluation of complex polynomials on equispaced arguments [10], error analysis [11], and a complexity analysis [12] are examples of research involving complex polynomials.

In the next section we describe the transformation which maps computation from the complex to the real domain. In Section 3 we show the complex E-method for polynomials. In Section 4 iterations and convergence conditions are considered. Implementation aspects are discussed in Section 
5.

\section{Complex-Real (CR) Transforms}

Complex numbers can be represented by $2 \times 2$ skewsymmetric matrices

$$
x+i y \leftrightarrow\left(\begin{array}{cc}
x & -y \\
y & x
\end{array}\right)
$$

This isomorphism holds for complex addition and multiplication which are used in the proposed method :

$$
\begin{aligned}
(a+i b)+(c+i d) & \leftrightarrow\left(\begin{array}{cc}
a & -b \\
b & a
\end{array}\right)+\left(\begin{array}{cc}
c & -d \\
d & c
\end{array}\right) \\
& =\left(\begin{array}{cc}
a+c & -b-d \\
b+d & a+c
\end{array}\right) \\
& \leftrightarrow(a+c)+i(b+d) \\
(a+i b) \times(c+i d) & \leftrightarrow\left(\begin{array}{cc}
a & -b \\
b & a
\end{array}\right) \times\left(\begin{array}{cc}
c & -d \\
d & c
\end{array}\right) \\
& =\left(\begin{array}{c}
a c-b d-(a d+b c) \\
a d+b c \\
a c-b d
\end{array}\right) \\
& \leftrightarrow(a c-b d)+i(b c+a d)
\end{aligned}
$$

Consequently, an $m \times n$ matrix of complex numbers can be represented as a $2 m \times 2 n$ matrix of real numbers. For $n \times n$ complex matrices, considered in this paper, the transform from the complex domain to the real domain is defined next.

Definition 1 The CR-transform of the n-dimensional complex linear system

$$
\left(\begin{array}{ccccc}
a_{1,1} & a_{1,2} & a_{1,3} & \cdots & a_{1, n} \\
a_{2,1} & a_{2,2} & a_{2,3} & \cdots & a_{2, n} \\
a_{3,1} & a_{3,2} & a_{3,3} & \cdots & a_{3, n} \\
\vdots & \vdots & \vdots & \cdots & \vdots \\
a_{n, 1} & a_{n, 2} & a_{n, 3} & \cdots & a_{n, n}
\end{array}\right) \times\left(\begin{array}{c}
z_{1} \\
z_{2} \\
z_{3} \\
\vdots \\
z_{n}
\end{array}\right)=\left(\begin{array}{c}
t_{1} \\
t_{2} \\
t_{3} \\
\vdots \\
t_{n}
\end{array}\right)
$$

is the $2 n$-dimensional real linear system $\left(\begin{array}{ccccccc}a_{1,1}^{r} & -a_{1,1}^{i} & a_{1,2}^{r} & -a_{1,2}^{i} & \cdots & a_{1, n}^{r} & -a_{1, n}^{i} \\ a_{1,1}^{i} & a_{1,1}^{r} & a_{1,2}^{i} & a_{1,2}^{r} & \cdots & a_{1, n}^{i} & a_{1, n}^{r} \\ a_{2,1}^{r} & -a_{2,1}^{i} & a_{2,2}^{r} & -a_{2,2}^{i} & \cdots & a_{2, n}^{r} & -a_{2, n}^{i} \\ a_{2,1}^{i} & a_{2,1}^{r} & a_{2,2}^{i} & a_{2,2}^{r} & \cdots & a_{2, n}^{i} & a_{2, n}^{r} \\ a_{3,1}^{r} & -a_{3,1}^{i} & a_{3,2}^{r} & -a_{3,2}^{i} & \cdots & a_{3, n}^{r} & -a_{3, n}^{i} \\ a_{3,1}^{i} & a_{3,1}^{r} & a_{3,2}^{i} & a_{3,2}^{r} & \cdots & a_{3, n}^{i} & a_{3, n}^{r} \\ \vdots & \vdots & \vdots & \vdots & \cdots & \vdots & \\ a_{n, 1}^{r} & -a_{n, 1}^{i} & a_{n, 2}^{r} & -a_{n, 2}^{i} & \cdots & a_{n, n}^{r} & -a_{n, n}^{i} \\ a_{n, 1}^{i} & a_{n, 1}^{r} & a_{n, 2}^{i} & a_{n, 2}^{r} & \cdots & a_{n, n}^{i} & a_{n, n}^{r}\end{array}\right) \times\left(\begin{array}{c}z_{1}^{r} \\ z_{1}^{i} \\ z_{2}^{r} \\ z_{2}^{i} \\ z_{3}^{r} \\ z_{3}^{i} \\ \vdots \\ z_{n}^{r} \\ z_{n}^{i}\end{array}\right)$ where $a_{j, k}=a_{j, k}^{r}+i a_{j, k}^{i}, z_{j}=z_{j}^{r}+i z_{j}^{i}$ and $t_{j}=t_{j}^{r}+i t_{j}^{i}$. These two linear systems are equivalent.

In other words, the real linear system (5) is obtained from the complex linear system (4) by replacing each element $x+i x$ by the $2 \times 2$ matrix defined in (1). In the next section we consider a hardware-oriented method for solving such a system.

\section{Complex E-method: An Overview}

The E-method [2, 3], provides an iterative approach of solving diagonally dominant real linear systems. The method has characteristics desirable for efficient hardware implementation: the basic operators are digit-vector multiplexers, redundant adders of $[p: 2]$ type, with $p \in\{3,4,6\}$ for radix-2, and registers. The overall structure consists of $n$ elementary units, interconnected digit-serially. The method computes one digit of each component of the solution per iteration in the MSDF (Most Significant Digit First) manner which allows digit-serial communication between the modules which operate concurrently. The time to obtain the solution to $m$ digits of precision is about $m$ cycles (iterations). The amount of hardware required is roughly related to the number of nonzero terms of the matrix of the system, which makes the E-method very efficient in hardware resources when the matrix of the system is sparse. Typical applications of the E-method are evaluation of polynomial and rational functions, since these correspond to sparse linear systems. The solution of the linear system

$$
\left(\begin{array}{ccccccc}
1 & -x & 0 & 0 & 0 & \cdots & 0 \\
0 & 1 & -x & 0 & 0 & \cdots & 0 \\
\vdots & \vdots & \vdots & \vdots & \vdots & \cdots & \vdots \\
0 & 0 & \cdots & 0 & 0 & 1 & -x \\
0 & 0 & 0 & \cdots & 0 & 0 & 1
\end{array}\right) \times\left(\begin{array}{l}
y_{0} \\
y_{1} \\
\vdots \\
y_{n-1} \\
y_{n}
\end{array}\right)
$$




$$
=\left(\begin{array}{l}
p_{0} \\
p_{1} \\
\vdots \\
p_{n-1} \\
p_{n}
\end{array}\right)
$$

is

$$
\left(\begin{array}{c}
p_{0}+p_{1} x+p_{2} x^{2}+\cdots+p_{n} x^{n} \\
p_{1}+p_{2} x+\cdots+p_{n} x^{n-1} \\
\vdots \\
p_{n-1}+p_{n} x \\
p_{n}
\end{array}\right)
$$

that is, the first component of the solution is

$$
p_{0}+p_{1} x+p_{2} x^{2}+\cdots+p_{n} x^{n}
$$

Now, let us turn to the evaluation of complex polynomials of a complex argument. We wish to evaluate

$$
p(z)=p_{0}+p_{1} z+p_{2} z^{2}+\ldots+p_{n} z^{n}
$$

where the $p_{j}$ 's and $z$ are complex numbers. As in the real case, the desired value $p(z)$ is clearly equal to the first component of the solution of the linear system

$$
\begin{gathered}
\left(\begin{array}{ccccccc}
1 & -z & 0 & 0 & 0 & \ldots & 0 \\
0 & 1 & -z & 0 & 0 & \ldots & 0 \\
0 & 0 & 1 & -z & 0 & \ldots & 0 \\
\vdots & \vdots & \vdots & \vdots & \vdots & \vdots & \vdots \\
0 & 0 & 0 & 0 & \ldots & 0 & 1
\end{array}\right) \times\left(\begin{array}{c}
y_{0} \\
y_{1} \\
y_{2} \\
y_{3} \\
y_{4} \\
\vdots \\
y_{n}
\end{array}\right) \\
=\left(\begin{array}{c}
p_{0} \\
p_{1} \\
p_{2} \\
p_{3} \\
p_{4} \\
\vdots \\
p_{n}
\end{array}\right)
\end{gathered}
$$

The E-method cannot directly solve the linear system (6), but now if we define real numbers $x$ and $y$ as $x+i y=z$, and $p_{j}^{r}$ and $p_{j}^{i}$ as $p_{j}=p_{j}^{r}+i p_{j}^{i}$, then we can apply the CRtransform to (6), and get the linear system with the coefficient matrix

$$
E=\left(\begin{array}{cccccccccc}
1 & 0 & -x & y & 0 & 0 & 0 & 0 & \cdots & 0 \\
0 & 1 & -y & -x & 0 & 0 & 0 & 0 & \cdots & 0 \\
0 & 0 & 1 & 0 & -x & y & 0 & 0 & \cdots & 0 \\
0 & 0 & 0 & 1 & -y & -x & 0 & 0 & \cdots & 0 \\
\vdots & \vdots & \vdots & \vdots & \vdots & \vdots & \vdots & \vdots & \vdots & \vdots \\
0 & 0 & \cdots & 0 & 0 & 0 & 1 & 0 & -x & y \\
0 & 0 & \cdots & 0 & 0 & 0 & 0 & 1 & -y & -x \\
0 & 0 & \cdots & 0 & 0 & 0 & 0 & 0 & 1 & 0 \\
0 & 0 & \cdots & 0 & 0 & 0 & 0 & 0 & 0 & 1
\end{array}\right)
$$

The first two components of the solution $s$ of the linear system

$$
E \times\left(\begin{array}{c}
s_{0}^{r} \\
s_{0}^{i} \\
s_{1}^{r} \\
s_{1}^{i} \\
\vdots \\
s_{n-1}^{r} \\
s_{n-1}^{i} \\
s_{n}^{r} \\
s_{n}^{i}
\end{array}\right)=\left(\begin{array}{c}
p_{0}^{r} \\
p_{0}^{i} \\
p_{1}^{r} \\
p_{1}^{i} \\
\vdots \\
p_{n-1}^{r} \\
p_{n-1}^{i} \\
p_{n}^{r} \\
p_{n}^{i}
\end{array}\right)
$$

are equal to the real and imaginary parts of

$$
p_{0}+p_{1} z+p_{2} z^{2}+\cdots+p_{n} z^{n} .
$$

For instance, in the case $n=3$, we get the solutions as shown in Figure 1. The linear system (7) is easily solved by the E-method, provided that it is diagonally dominant. The iterations and convergence conditions are discussed in the next section. Note that the E-method does not evaluate directly the expressions given for the solution $s_{0}$. These would require at least $16+16$ full-precision multiplications which, assuming enough multipliers are available, would take at least 3 consecutive multiply times. Moreover, the reduction of product terms would require a [10:2] reduction. Of course, all the interconnections are of full precision. Instead, as explained later, the complex E-method computes $s_{0}$ on 14 serial-parallel (left-to-right) multipliers, including the additions, in about one serial-parallel multiplication time. In this approach, the interconnections are digit-serial. 


$$
s=\left(\begin{array}{c}
-3 x y^{2} p_{3}^{r}+x^{3} p_{3}^{r}-3 y x^{2} p_{3}^{i}+x^{2} p_{2}^{r}-2 x y p_{2}^{i}+x p_{1}^{r}+y^{3} p_{3}^{i}-y^{2} p_{2}^{r}-y p_{1}^{i}+p_{0}^{r} \\
-y^{3} p_{3}^{r}+3 y x^{2} p_{3}^{r}-3 y^{2} x p_{3}^{i}+2 y x p_{2}^{r}-y^{2} p_{2}^{i}+y p_{1}^{r}+x^{3} p_{3}^{i}+x^{2} p_{2}^{i}+x p_{1}^{i}+p_{0}^{i} \\
-y^{2} p_{3}^{r}+x^{2} p_{3}^{r}-2 y x p_{3}^{i}+x p_{2}^{r}-y p_{2}^{i}+p_{1}^{r} \\
x^{2} p_{3}^{i}+2 x y p_{3}^{r}-y^{2} p_{3}^{i}+y p_{2}^{r}+x p_{2}^{i}+p_{1}^{i} \\
x p_{3}^{r}-y p_{3}^{i}+p_{2}^{r} \\
y p_{3}^{r}+x p_{3}^{i}+p_{2}^{i} \\
p_{3}^{r} \\
p_{3}^{i}
\end{array}\right)
$$

Figure 1. Solutions to system (7)

\section{Iteration, and convergence conditions}

To make the presentation simpler, we will focus on radix2 iterations only. Adaptation to higher radices is rather straightforward. The radix-2 E-method consists in solving the $n$-dimensional linear system

$$
A x=P
$$

by using the following basic recursion on residuals:

$$
w^{(j)}=2 \times\left[w^{(j-1)}-A d^{(j-1)}\right]
$$

with $w^{(0)}=\left[p_{0}, p_{1}, \ldots, p_{n}\right]^{t}$, and $d^{(j)}=\left[d_{0}, d_{1}, \ldots, d_{n}\right]^{t}$ where the digits $d_{k}^{(j)}$ are in $\{-1,0,1\}$. Define the number $D_{k}^{(j)}=d_{k}^{(0)} \cdot d_{k}^{(1)} d_{k}^{(2)} \ldots d_{k}^{(j)}$ (the $d_{k}^{(j)}$ are the digits of a radix-2 signed-digit representation of $\left.D_{k}^{(j)}\right)$. By induction, we easily get,

$$
w^{(j)}=2^{j}\left[w^{(0)}-A D^{(j-1)}\right] .
$$

Using (9), one can show that if the residuals $\left|w_{k}^{(j)}\right|$ are bounded, then for all $k, D_{k}^{(j)}$ goes to $y_{k}$ as $j$ goes to infinity.

The problem at step $j$ is to find a selection function that gives a value of the digits $d_{k}^{(j)}$ from the residuals $w_{k}^{(j)}$ such that the values $w_{k}^{(j+1)}$ will remain bounded. In [3], the following selection function (a form of rounding) is proposed

$$
s(x)=\left\{\begin{array}{lr}
\operatorname{sign} x \times\lfloor|x+1 / 2|\rfloor, & \text { if }|x| \leq 1 \\
\operatorname{sign} x \times\lfloor|x|\rfloor, & \text { otherwise, }
\end{array}\right.
$$

and applied to the following cases:

1. $d_{k}^{(j)}=s\left(w_{k}^{(j)}\right)$, i.e., the selection uses a non-redundant $w_{k}^{(j)}$;
2. $d_{k}^{(j)}=s\left(\hat{w}_{k}^{(j)}\right)$, where $\hat{w}_{k}^{(j)}$ is an approximation to $w_{k}^{(j)}$ (in practice, $\hat{w}_{k}^{(j)}$ is deduced from a few digits of $w_{k}^{(j)}$ by the means of a rounding or a truncation)

Let us now consider in more detail complex polynomial evaluation. We wish to evaluate a degree- $n$ polynomial

$$
p_{n} z^{n}+p_{n-1} z^{n-1}+\cdots+p_{0}
$$

at the complex point $z=x+i y$, with $p_{k}=p_{k}^{r}+i p_{k}^{i}$. The matrix of the CR-transform, obtained in Section 3 is

$$
A=\left(\begin{array}{cccccccccc}
1 & 0 & -x & y & 0 & 0 & 0 & 0 & \cdots & 0 \\
0 & 1 & -y & -x & 0 & 0 & 0 & 0 & \cdots & 0 \\
0 & 0 & 1 & 0 & -x & y & 0 & 0 & \cdots & 0 \\
0 & 0 & 0 & 1 & -y & -x & 0 & 0 & \cdots & 0 \\
\vdots & \vdots & \vdots & \vdots & \vdots & \vdots & \vdots & \vdots & \vdots & \vdots \\
0 & \cdots & 0 & 0 & 0 & 0 & 1 & 0 & -x & y \\
0 & \cdots & 0 & 0 & 0 & 0 & 0 & 1 & -y & -x \\
0 & \cdots & 0 & 0 & 0 & 0 & 0 & 0 & 1 & 0 \\
0 & \cdots & 0 & 0 & 0 & 0 & 0 & 0 & 0 & 1
\end{array}\right)
$$

Let us slightly modify the notations $w$ and $d$ of iteration (8), to adapt them to the complex case. The residual vector $w^{(j)}$ will be denoted

$$
w^{(j)}=\left[w_{0, r}^{(j)}, w_{0, i}^{(j)}, w_{1, r}^{(j)}, w_{1, i}^{(j)}, \cdots, w_{n, r}^{(j)}, w_{n, i}^{(j)}\right],
$$

and its initial value will be given by

$$
\left\{\begin{array}{l}
w_{k, r}^{(0)}=p_{k}^{r} \\
w_{k, i}^{(0)}=p_{k}^{i}
\end{array}\right.
$$


The digit-vector $d^{(j)}$ will be denoted

$$
d^{(j)}=\left[d_{0, r}^{(j)}, d_{0, i}^{(j)}, d_{1, r}^{(j)}, d_{1, i}^{(j)}, \cdots, d_{n, r}^{(j)}, d_{n, i}^{(j)}\right] .
$$

Therefore, iteration (8) becomes

- for $k=0, \ldots, n-1$,

$$
\left\{\begin{array}{l}
w_{k, r}^{(j)}=2\left[w_{k, r}^{(j-1)}-d_{k, r}^{(j-1)}+x d_{k+1, r}^{(j-1)}-y d_{k+1, i}^{(j-1)}\right] \\
w_{k, i}^{(j)}=2\left[w_{k, i}^{(j-1)}-d_{k, i}^{(j-1)}+y d_{k+1, r}^{(j-1)}+x d_{k+1, i}^{(j-1)}\right]
\end{array}\right.
$$

- for $k=n$,

$$
\left\{\begin{array}{l}
w_{n, r}^{(j)}=2\left[w_{n, r}^{(j-1)}-d_{n, r}^{(j-1)}\right] \\
w_{n, i}^{(j)}=2\left[w_{n, i}^{(j-1)}-d_{n, i}^{(j-1)}\right]
\end{array}\right.
$$

Now, let us examine the convergence conditions. The iterations converge to the desired result if vector $w^{(j)}$ is bounded. Define constants $\xi, \alpha$ and $\Delta$ (with $0 \leq \Delta<1$ ) such that

1. $|x|+|y| \leq \alpha$

2. for any $k$ between 0 and $n$,

$$
\begin{cases}\left|p_{k}^{r}\right| & \leq \xi \\ \left|p_{k}^{i}\right| & \leq \xi \\ \left|w_{k, r}^{(j)}-\hat{w}_{k, r}^{(j)}\right| & \leq \frac{\Delta}{2} \\ \left|w_{k, i}^{(j)}-\hat{w}_{k, i}^{(j)}\right| & \leq \frac{\Delta}{2}\end{cases}
$$

Since $\left|d_{k, r}^{(j-1)}-\hat{w}_{k, r}^{(j-1)}\right| \leq 1 / 2$ and $\left|d_{k, i}^{(j-1)}-\hat{w}_{k, i}^{(j-1)}\right| \leq 1 / 2$, from (11) we find

$$
\left|w_{k, r}^{(j)}\right| \leq 2\left(\frac{1}{2}+\frac{\Delta}{2}+\alpha\right)=1+\Delta+2 \alpha .
$$

The same bound holds for $\left|w_{k, i}^{(j)}\right|$. For this bound to be feasible, we must assure that a suitable choice of $d_{k, r}^{(j)}$ and $d_{k, r}^{(j)}$ in $\{-1,0,1\}$ is possible. This requires that $\left|w_{k, r}^{(j)}\right|$ and $\left|w_{k, i}^{(j)}\right|$ should be less than $3 / 2$. This immediately gives the following condition

$$
\Delta+2 \alpha \leq \frac{1}{2} .
$$

Now, let us turn to the initial values. Since $\left|w_{k, r}^{(0)}\right|$ and $\left|w_{k, i}^{(0)}\right|$ must also be less than $3 / 2$, we get

$$
\xi \leq \frac{3}{2}
$$

Consider the following example: we wish to evaluate

$$
p(z)=(1+i) z^{3}-(0.5+1.25 i) z^{2}+z+1 .
$$

at point

$$
z=\frac{1}{100}+\frac{i}{10}
$$

We assume that $\Delta=0$ (that is, we use non-redundant residuals). We get:

- Initialization:

$$
\begin{aligned}
w^{(0)} & =\left[p_{0}^{r}, p_{0}^{i}, p_{1}^{r}, p_{1}^{i}, p_{2}^{r}, p_{3}^{i}, p_{4}^{r}, p_{4}^{i}\right]^{t} \\
& =[1,0,1,0,-0.5,-1.25,1,1]^{t}
\end{aligned}
$$

- Step 1: from $w^{(0)}$ and the selection function, we get

$$
s^{(0)}=[1,0,1,0,0,-1,1,1]^{t},
$$

which gives

$$
w^{(1)}=[0.02,0.2,0.2,-0.02,-1.18,-0.28,0,0]^{t} .
$$

- Step 2: from $w^{(1)}$ and the selection function, we get

$$
s^{(1)}=[0,0,0,0,-1,0,0,0]^{t},
$$

which gives

$$
w^{(2)}=[0.04,0.4,0.38,-0.24,-0.36,-0.56,0,0]^{t} .
$$

- After 20 iterations, the number

$$
d_{0, r}^{(0)} \cdot d_{0, r}^{(1)} d_{0, r}^{(2)} \cdots d_{0, r}^{(20)}+i \times d_{0, i}^{(0)} \cdot d_{0, i}^{(1)} d_{0, i}^{(2)} \cdots d_{0, i}^{(20)}
$$

is equal to

$$
\begin{aligned}
& \frac{533789}{524288}+\frac{57727}{524288} i \approx 1.018121719+0.110105514 i \\
& \text { whereas the exact value of } p(z) \text { is }
\end{aligned}
$$

$$
p(z)=1.018121+0.110106 i
$$

Exactly as in the real case, even if polynomial $p$ and point $z$ do not satisfy the convergence constraints, one can easily "transform" them using mere shifts, so that $p(z)$ can be computed using the E-method. Once $\Delta$ is chosen, and $\alpha$ is defined as $\frac{1}{4}-\Delta / 2$, this is done as follows:

1. Find the smallest integer $k$ such that $\left|\Re\left(z / 2^{k}\right)\right|+$ $\left|\Im\left(z / 2^{k}\right)\right|$ should be less than $\alpha$;

2. Now, $p(z)=\pi(t)$, where the degree- $m$ coefficient of polynomial $\pi$ is $2^{m k} p_{m}$. If at least one of the coefficients of $\pi$ has the absolute value of its real or imaginary part greater than $\xi=3 / 2$, then divide $\pi$ by $2^{\ell}$, where $\ell$ is the smallest integer such that $\rho=\pi / 2^{\ell}$ has the absolute value of the real and imaginary parts of its coefficients less than $\xi$;

3. What we actually compute using the E-method is $\rho\left(z / 2^{k}\right)$. This result will then be multiplied by $2^{\ell}$ (a simple left-shift) to get $p(z)$. 


\subsection{Implementation of the Proposed Method}

In this section we discuss implementation of the complex E-method for polynomials. The main difference from implementation of real domain E-method is that the number of non-zero off-diagonal elements doubles: for the polynomial case to two. This has two consequences. First, the bounds on the elements are smaller by a factor of two, and second, the cycle time is increased as explained later in this section. The corresponding implementations considered for the real domain E-method are in [2, 3, 7].

A general scheme for evaluation of complex polynomials is shown in Figure 2 for $n=3$ and the corresponding elementary unit (PEU) is illustrated in Figure 3. A bit-parallel bus transmits $x$ and $y$ values in a broadcast mode, while the real and imaginary coefficients $p^{r}$ and $p^{i}$ are loaded in separate cycles. Note that the initialization cycles could be shorter than the iteration cycles.

A block diagram of an Elementary Unit for polynomial evaluation $(P E U)$ is shown in Figure 3.

The modules in Figure 3 are:

- Registers (4)

- Multiple generators MG (2), producing $\{-1,0,1\} \times x$ and $\{-1,0,1\} \times y$, with buffers

- Multiplexer MUX for initializing the residual

- A [4:2] adder

- Output digit selection $S E L$ ( a table or a gate network)

The digit-serial outputs of $P E U 0$ can be converted into digit-parallel form using on-the-fly converters $O F C$ [7]. The cycle time, in terms of a full adder (complex gate) delay $t$, is estimated as

$$
\begin{aligned}
T_{P E U} & =t_{B U F F}+t_{M G}+t_{S E L}+t_{[4: 2]}+t_{R E G} \\
& \approx(0.4+0.3+1+1.3+0.9) t=3.9 t
\end{aligned}
$$

The cost, again in terms of area of a full adder $A_{F A}$, is estimated as

$$
\begin{aligned}
A_{P E U}(m) & =A_{S E L}+2 A_{B U F F}+2 A_{M G} \\
& +A_{M U X}+A_{[4: 2]}+4 A_{R E G}+2 A_{O F C} \\
& \approx[5+2 \times 0.4+(m+2)(3 \times 0.45 \\
& +2.3+4 \times 0.6+2 \times 2.1)] A_{F A} \\
& \approx 16+10 m A_{F A}
\end{aligned}
$$

The cost is measured as area occupied by modules using the area of a full-adder $\left.A_{F A}\right)$ as the unit. The area of primitive modules is given in Table 1 .

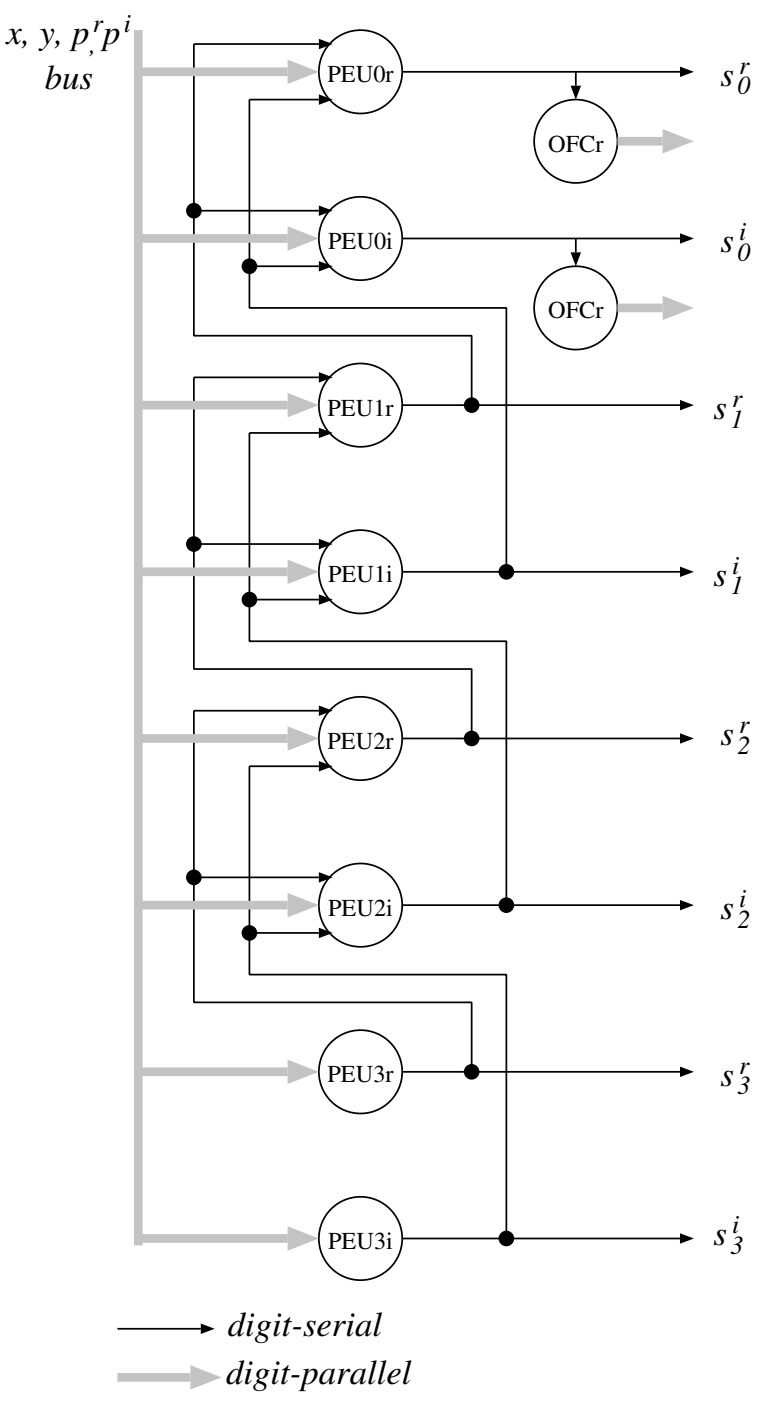

Figure 2. Overall scheme for evaluating complex polynomial of degree $n=3$. 


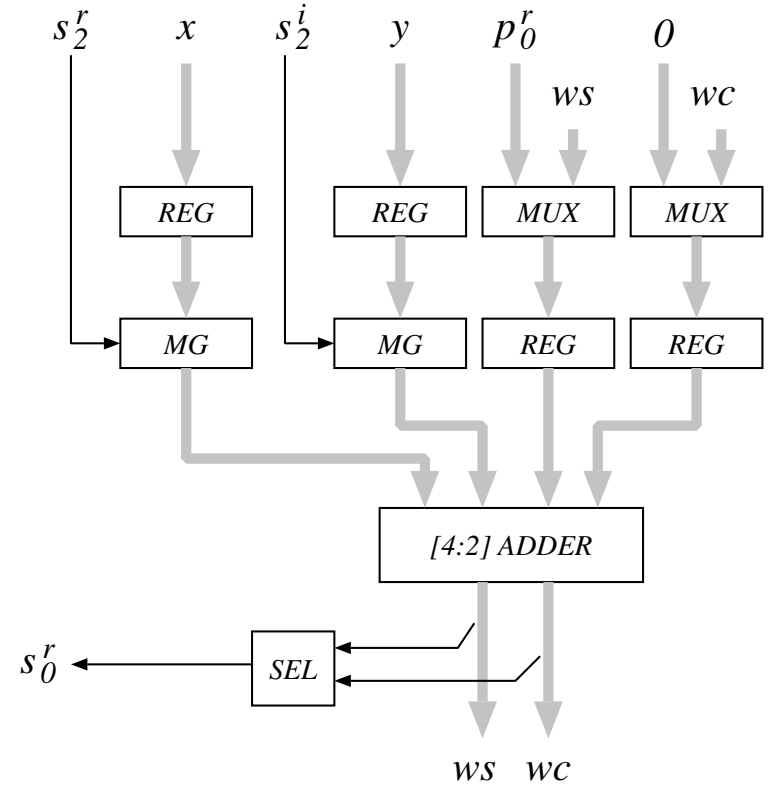

Figure 3. Block diagram of Elementary Unit for polynomials $\left(P E U_{0}\right.$.)

Table 1. Area of primitive modules (in $A_{F A}$ units).

\begin{tabular}{|l|l|}
\hline Module[1bit $]$ & Area $\left[A_{F A}\right]$ \\
\hline \hline Register & $A_{R E G}=0.6$ \\
\hline Buffer & $A_{B U F F}=0.4$ \\
\hline$M U X$ & $A_{M U X}=0.45$ \\
\hline$M G$ & $A_{M G}=0.45$ \\
\hline$[4: 2]$ adder & $A_{[4: 2]}=2.3$ \\
\hline$S E L$ & $A_{S E L}=5$ \\
\hline On-the-fly converter & $A_{O F C}=2 A_{M U X}+2 A_{R E G}=2.1$ \\
\hline
\end{tabular}

[1] K. Benmahammed, Evaluation of Complex Polynomials in One and Two Variables. Multidimensional Systems and Signal Processing, 5, 245-261, 1994. 
[2] M.D. Ercegovac. A general method for evaluation of functions and computation in a digital computer. $\mathrm{PhD}$ thesis, Dept. of Computer Science, University of Illinois, Urbana-Champaign, 1975.

[3] M.D. Ercegovac. A general hardware-oriented method for evaluation of functions and computations in a digital computer. IEEE Trans. Comp., C-26(7):667-680, 1977.

[4] M.D. Ercegovac and J.-M. Muller. Complex Division with Prescaling of Operands. IEEE International Conference on Application-Specific Systems, Architectures and Processors, pp. 293-303, 2003.

[5] M.D. Ercegovac and J.-M. Muller, Design of a complex divider. Proc. SPIE on Advanced Signal Processing Algorithms, Architectures, and Implementations XII, pp. 51-59, 2004.

[6] M.D. Ercegovac and J.-M. Muller. Complex Square Root with Operand Prescaling. IEEE International Conference on Application-Specific Systems, Architectures and Processors, pp. 293-303, 2004.

[7] M.D. Ercegovac and T. Lang. Digital Arithmetic, Morgan Kaufmann Publishers - an Imprint of Elsewier Science, San Francisco, 2004.

[8] M.D. Ercegovac and J.-M. Muller, Solving Systems of Linear Equations in Complex Domain : Complex E-Method. LIP Report No. 2007-2, École Normale Supérieure de Lyon, France.

[9] N. Brisebarre and J.-M. Muller. Functions approximable by E-fractions. 38th Asilomar Conference on Signals, Systems and Computers, Pacific Grove, California, Nov. 2004.

[10] A.H. Nutall, Efficient Evaluation of Polynomials and Exponentials of Polynomials for Equispaced Arguments, IEEE Trans. Acoust., Speech, Signal Process., vol. ASSP-35, pp. 1486-1487, 1987.

[11] F. W. J. Olver, Error Bounds for Polynomial Evaluation and Complex Arithmetic, IMA Journal of $\mathrm{Nu}-$ merical Analysis 6, 373-379, 1986.

[12] J.H. Reif, Approximate Complex Polynomial Evaluation in Near Constant Work Per Point, STOC 97, pp. 30-39, 1997. 